\title{
Systemic inflammatory indices predict tumor response to neoadjuvant chemoradiotherapy for locally advanced rectal cancer
}

\author{
SICONG LAI ${ }^{1,2^{*}}$, LIANG HUANG ${ }^{1,2^{*}}$, SHUANGLING LUO ${ }^{1,2}$, ZHANZHEN LIU ${ }^{1,2}$, \\ JIANGHUI DONG ${ }^{3}$, LIPING WANG ${ }^{3}$ and LIANG KANG ${ }^{1,2}$
}

\author{
${ }^{1}$ Department of Colorectal Surgery; ${ }^{2}$ Guangdong Institute of Gastroenterology, Guangdong Provincial Key Laboratory of \\ Colorectal and Pelvic Floor Diseases, The Sixth Affiliated Hospital of Sun Yat-sen University, Guangzhou, \\ Guangdong 510655, P.R. China; ${ }^{3}$ UniSA Clinical and Health Science, UniSA Cancer \\ Research Institute, University of South Australia, Adelaide, SA 5001, Australia
}

Received March 13, 2020; Accepted June 17, 2020

DOI: $10.3892 / \mathrm{ol} .2020 .11812$

\begin{abstract}
Systemic inflammatory responses are associated with the prognosis of patients with colorectal cancer. However, the value in predicting tumor responses to neoadjuvant chemoradiotherapy (nCRT) remains to be elucidated. The current study aimed to investigate the association between systemic inflammatory indices and pathological complete response $(\mathrm{pCR})$. The training and validation cohorts included 225 and 96 patients with locally advanced rectal cancer. The neutrophil-to-lymphocyte ratio (NLR) and platelet-to-lymphocyte ratio were recorded prior to nCRT and radical surgery. Univariate and multivariate analysis were used to investigate the association between systemic inflammatory indices and pCR. Systemic inflammatory indices prior to or following
\end{abstract}

Correspondence to: Dr Liping Wang, UniSA Clinical and Health Science, UniSA Cancer Research Institute, University of South Australia, HB Building Cnr North Terrace, Morphett Street, Adelaide, SA 5001, Australia

E-mail: liping.wang@mymail.unisa.edu.au

Professor Liang Kang, Department of Colorectal Surgery, Guangdong Provincial Key Laboratory of Colorectal and Pelvic Floor Diseases, The Sixth Affiliated Hospital of Sun Yat-sen University, 26 Yuancun Erheng Road, Guangzhou, Guangdong 510655, P.R. China

E-mail:kangl@mail.sysu.edu.cn

*Contributed equally

Abbreviations: nCRT, neoadjuvant chemoradiotherapy; LARC, locally advanced rectal cancer; NLR, neutrophil-to-lymphocyte ratio; PLR, platelet-to-lymphocyte ratio; pCR, pathological complete response; TRG, tumor regression grade; CEA, carcino-embryonic antigen

Key words: local advanced rectal cancer, neoadjuvant chemoradiotherapy, systemic inflammatory indices, colorectal cancer, pathological complete response treatment had no significant association with pCR; however, the percentage change in NLR from pre-nCRT to post-nCRT was associated with a poor response, and a percentage change of $>21.5 \%$ NLR $(\mathrm{P}=0.006 ; \mathrm{OR}=0.413 ; 95 \% \mathrm{CI}=0.22-0.773)$ was a predictor of poor $\mathrm{pCR}$. Therefore, in rectal cancer, the percentage change in NLR from pre- to post-nCRT was found to be a predictor of poor $\mathrm{pCR}$.

\section{Introduction}

Colorectal cancer has become one of the most common tumors and is the third most common malignancy. Globally, $\sim 1.2$ million patients are diagnosed with colorectal cancer and $>600,000$ die from the disease in 2008 (1). Neoadjuvant chemoradiotherapy (nCRT) prior to radical surgery and post-operative adjuvant chemotherapy is the standard treatment for locally advanced rectal cancer (LARC) $(2,3)$. Certain complications can arise from radical surgery that decrease quality of life, including anastomotic leakage, anastomotic stenosis, urinary and/or sexual dysfunction, low anterior resection syndrome and increased probability of enterostomy (4-6).

Response to neoadjuvant therapy is heterogeneous and $\sim 10-30 \%$ of patients with colorectal cancer achieve pathological complete response (pCR) (7). The 'watch and wait' strategy and local resection for patients with pCR following nCRT has become a focus of study $(8,9)$. Generally, pCR is predicted through digital rectum examination, imaging examination, endoscopy and tumor-related markers (9-12). However, the predictions made using these techniques are not always in line with the pathology results $(8,13)$. Currently, there are no affordable and reliable markers that can predict tumor response.

Systemic inflammatory responses have been reported to be predictors of prognosis for numerous types of solid tumors, including gastrointestinal $(14,15)$, pancreatic (16), renal (17) and breast cancer (18). Systemic inflammatory responses can be reflected by hematologic parameters, including lymphocyte count and platelet count, or the ratio of different cell 
types, including the platelet-to-lymphocyte ratio (PLR) and neutrophil-to-lymphocyte ratio (NLR) $(19,20)$. The tumor microenvironment is closely associated with the occurrence and development of cancer (21). In the peripheral circulation, neutrophils and lymphocytes act as immunocytes, reflecting the tumor microenvironment, and the number of immunocytes changes according to patient age and immunoreactivity (22). Changes in the NLR may be associated with changes in tumor size during regression due to the nCRT for rectal cancers (23). However, it remains unclear whether systematic inflammatory responses could be used as a marker for identifying patients with tumors achieving pCR following nCRT.

Therefore, the current study investigated pre- and post-nCRT systemic inflammatory indices and changes in these indices from pre-nCRT to post-nCRT to determine the association between these parameters and responsiveness to nCRT in patients with LARC.

\section{Materials and methods}

Patients. Data from patients with rectal cancer who received nCRT and radical surgery at the Sixth Affiliated Hospital between January 2013 and October 2018, were retrospectively analyzed. A total of 321 patients were included in this study. The mean age of patients was 58.5 years. A total of 218 patients were male $(67.9 \%)$ and 103 patients were female (32.1\%). The inclusion criteria were as follows: i) 18-75 years old; ii) diagnosis of rectal adenocarcinoma confirmed by biopsy pathology; iii) stage II (T3/T4; N0M0) or stage III (T1-4; $\mathrm{N}^{+} \mathrm{M} 0$ ) evaluated by thoracic and abdominal pelvic CT, pelvic MRI or transluminal ultrasound prior to nCRT; iv) the distance from the anal verge evaluated by digital rectum examination or enteroscopy was $<12 \mathrm{~cm}$; v) an Eastern Cooperative Oncology Group score of 0-2; vi) (24) no radiotherapy, chemotherapy or surgical contraindications; and vii) no past medical history of malignant tumors. The exclusion criteria included the following: i) Absence of clinical data; ii) infections prior to new adjuvant therapy or surgery; iii) use of leukocyte enhancing drugs; and iv) incomplete new adjuvant therapy or radical surgery. Prior to the study, all patients provided written informed consent. The current study was approved by the Institutional Review Board of the Sixth Affiliated Hospital following rigorous review.

Treatment. All patients were assessed by a multiple disciplinary team at The Sixth Affiliated Hospital of Sun Yat-sen University and underwent nCRT and radical surgery. The total dose of radiotherapy given was $46-50.4$, or $1.8-2.0 \mathrm{~Gy}$ for 23-28 fractions. Patients received one of the following two chemotherapy regimens: mFOLFOX6 [85 mg/m² oxaliplatin, $400 \mathrm{mg} / \mathrm{m}^{2}$ leucovorin and $400 \mathrm{mg} / \mathrm{m}^{2}$ fluorouracil $(5-\mathrm{FU})$ administered through intravenous drip on day 1 , followed by $2,400 \mathrm{mg} / \mathrm{m}^{2}$ fluorouracil continuously administered through intravenous infusion for $48 \mathrm{~h}$ ] or DeGramont $\left(400 \mathrm{mg} / \mathrm{m}^{2}\right.$ leucovorin, $400 \mathrm{mg} / \mathrm{m}^{2} \mathrm{~F}-5 \mathrm{U}$ administered through intravenous drip on day 1 , followed by $2,400 \mathrm{mg} / \mathrm{m}^{2}$ fluorouracil continuously administered through intravenous infusion for $48 \mathrm{~h}$ ). The interval between radiotherapy and surgery was 4-12 weeks and the interval between chemotherapy and surgery was 2-4 weeks.
Peripheral blood examinations. Peripheral blood $(2 \mathrm{ml})$ was collected from all patients within 1 week prior to the first nCRT (pre-nCRT) and 1 week prior to surgery (post-nCRT). Neutrophil, lymphocyte and platelet counts were obtained from the hospital information system. The PLR was calculated as the absolute count of platelets/lymphocytes and NLR was calculated as the absolute count of neutrophils/lymphocytes. The percentage change in NLR from pre-nCRT to post-nCRT was defined as [(post-NLR-pre-NLR)/pre-NLR x100\%]. A result of $\geq 0$ indicated that NLR following new adjuvant treatment was increased, whereas a result of $<0$ indicated that NLR after new adjuvant treatment was decreased. The percentage change in PLR from pre-nCRT to post-nCRT was defined as [(post-PLR-pre-PLR)/pre-PLR x100\%].

Pathological assessment. Two experienced pathologists evaluated the tumor regression grades (TRG) of patients based on the American Joint Committee on Cancer (AJCC; 8th edition) (25) as follows: 0, no viable cancer cells (complete response); 1 , single cells or rare small groups of cancer cells (near-complete response); 2, residual cancer with evident tumor regression and with single cells or rare small groups of cancer cells (partial response); and 3, extensive residual cancer with no evident tumor regression (poor or no response), pCR was defined as no signs of viable cancer cells in resected specimens and in the lymph nodes.

Statistical analysis. SPSS software (version 24.0; IBM Corp.) was used to analyze data. $\mathrm{P}<0.05$ was considered to indicate a statistically significant difference. The $\chi^{2}$ test was used to compare the differences between the qualitative variables of the cohorts. The Shapiro-Wilk test confirmed that the quantitative data did not conform to a normal distribution. Therefore, quantitative data between cohorts were compared by the Mann-Whitney U test. Tumor patients were strictly divided into a pCR group (TRG 0) and a non-pCR group (TRG 1-3) according to TRG stages. The 'cut-off' value of the indices was determined by the receiver operating characteristic curve (ROC). Parameters were compared using the $\chi^{2}$ and t-tests between pCR and non-pCR groups. The association between systemic inflammatory indices and pCR were analyzed by univariate and multivariate logistic regression analysis. The multivariate analysis included the variables of $\mathrm{P}<0.1$ in the univariate analysis.

\section{Results}

Patient characteristics. Baseline characteristics of patients in the two cohorts are presented in Table 1. In the training cohort, a total of 152 patients were male (67.6\%) and 73 patients were female $(32.4 \%)$. A total of $162(72 \%)$ patients received a chemotherapy regimen with oxaliplatin (mFOLFOX6). Furthermore, 69 patients (30.6\%) achieved pCR. In the validation cohort, 66 patients $(68.7 \%)$ were male and 30 patients $(31.3 \%)$ were female. A total of 67 patients $(69.8 \%)$ received chemotherapy regimens with oxaliplatin. There were 26 patients $(27.1 \%)$ who achieved pCR. No statistical differences were observed in clinical characteristics, treatment factors and systemic inflammatory indices between the two cohorts $(\mathrm{P}>0.05)$. 
Table I. Baseline characteristics of the patients in the training and validation cohorts.

\begin{tabular}{|c|c|c|c|}
\hline Variable & Training cohort & Validation cohort & P-value \\
\hline Age, years & & & 0.670 \\
\hline$\leq 65$ & 183 & 80 & \\
\hline$>65$ & 42 & 16 & \\
\hline Sex & & & 0.834 \\
\hline Male & 152 & 66 & \\
\hline Female & 73 & 30 & \\
\hline CEA prior to treatment, $\mu \mathrm{g} / \mathrm{l}$ & & & 0.621 \\
\hline$\leq 5$ & 134 & 60 & \\
\hline$>5$ & 91 & 36 & \\
\hline Clinical T stage & & & 0.977 \\
\hline $\mathrm{T} 2$ & 8 & 3 & \\
\hline $\mathrm{T} 3$ & 167 & 72 & \\
\hline $\mathrm{T} 4$ & 50 & 21 & \\
\hline Clinical N stage & & & 0.574 \\
\hline No & 32 & 16 & \\
\hline $\mathrm{N} 1-2$ & 193 & 80 & \\
\hline Tumor size, $\mathrm{cm}$ & & & 0.126 \\
\hline$\leq 5$ & 173 & 66 & \\
\hline$>5$ & 52 & 30 & \\
\hline Tumor circumference, $\%$ & & & 0.838 \\
\hline$\leq 50$ & 91 & 40 & \\
\hline$>50$ & 134 & 56 & \\
\hline Mesorectal fascia & & & 0.659 \\
\hline Positive & 76 & 30 & \\
\hline Negative & 149 & 66 & \\
\hline Distance from anal verge, $\mathrm{cm}$ & & & 0.280 \\
\hline$\leq 5$ & 126 & 60 & \\
\hline$>5$ & 99 & 36 & \\
\hline Tumor differentiation & & & 0.929 \\
\hline High & 71 & 8 & \\
\hline Medium & 133 & 56 & \\
\hline Low & 21 & 32 & \\
\hline Operation interval of radiotherapy, weeks & & & 0.178 \\
\hline$\leq 7$ & 93 & 32 & \\
\hline$>7$ & 132 & 64 & \\
\hline Chemotherapy regimens & & & 0.689 \\
\hline With oxaliplatin & 162 & 67 & \\
\hline Without oxaliplatin & 63 & 29 & \\
\hline Chemotherapy courses & & & 0.055 \\
\hline$\leq 4$ & 30 & 21 & \\
\hline$>4$ & 195 & 75 & \\
\hline Tumor response & & & 0.520 \\
\hline $\mathrm{pCR}$ & 69 & 26 & \\
\hline Non-pCR & 156 & 70 & \\
\hline Mean value of pre-NLR (interval) & $2.39(0.59-17.04)$ & $2.52(0.79-27.46)$ & 0.626 \\
\hline Mean value of pre-PLR (interval) & $148.57(40.31-536.00)$ & $144.21(47.92-448.84)$ & 0.596 \\
\hline Mean value of post-NLR (interval) & $3.66(0.81-16.79)$ & $3.09(0.77-12.99)$ & 0.078 \\
\hline Mean value of post-PLR (interval) & $237.63(77.25-1007.07)$ & $220.87(84.11-595.30)$ & 0.556 \\
\hline Mean value of percentage change in NLR, \% (interval) & $80(-81-1066)$ & $57(-91-412)$ & 0.179 \\
\hline Mean value of percentage change in PLR, \% (interval) & $74(-73-605)$ & $69(-60-343)$ & 0.614 \\
\hline
\end{tabular}

CEA, carcino-embryonic antigen; T, tumor; N, lymph node; pCR, pathological complete response; NLR, neutrophil-to-lymphocyte ratio; PLR, platelet-to-lymphocyte ratio. 
Table II. Association between systemic inflammatory indices and $\mathrm{pCR}$ in the training cohort.

\begin{tabular}{lccc}
\hline Variable (mean) & $\begin{array}{c}\text { pCR Group } \\
(\mathrm{n}=69)\end{array}$ & $\begin{array}{c}\text { Non-pCR Group } \\
(\mathrm{n}=156)\end{array}$ & P-value \\
\hline Pre-NLR & 2.55 & 2.32 & 0.234 \\
Pre-PLR & 157.30 & 144.71 & 0.302 \\
Post-NLR & 3.34 & 3.80 & 0.071 \\
Post-PLR & 232.40 & 239.94 & 0.761 \\
\% change in NLR & 63 & 87 & 0.036 \\
\% change in PLR & 61 & 80 & 0.170 \\
\hline
\end{tabular}

pCR, pathological complete response; NLR, neutrophil-to-lymphocyte ratio; PLR, platelet-to-lymphocyte ratio.

Analysis for $p C R$ in the training cohort. Associations between systemic inflammation indices and pCR for the training cohort are presented in Table II. The mean value of the percentage change in NLR was $63 \%$ in the pCR group and $87 \%$ in the non-pCR group. Additionally, the percentage change in NLR was significantly increased in the $\mathrm{pCR}$ group compared with the non-pCR group ( $\mathrm{P}=0.036$; Fig. 1). However, there were no significant differences in the other pre- and post-treatment systemic inflammation indices between the pCR and non-pCR group.

The area under the curve of the ROC analysis was 0.588 $(\mathrm{P}=0.036$; Fig. 2). Therefore, we divided the percentage change in NLR into two categories for subsequent analyses: A percentage change of $\leq 21.5 \%$ NLR and a percentage change of $>21.5 \%$ NLR.

Univariate analysis demonstrated that carcino-embryonic antigen (CEA) prior to treatment, tumor differentiation, chemotherapy regimens and the percentage change in NLR were associated with pCR (Table III). A total of $45 \%$ of patients with a percentage change of $\leq 21.5 \%$ NLR achieved pCR compared with $23 \%$ of patients with a percentage change of $>21.5 \%$ NLR ( $\mathrm{P}=0.001$; Fig. 3). Multivariate analysis included all variables in the univariate analysis, including sex, CEA prior to treatment, tumor scope, tumor differentiation, chemotherapy regimens and the percentage change in NLR $(\mathrm{P}<0.1)$. However, only the percentage change in NLR $(\mathrm{P}=0.006$; $\mathrm{OR}=0.413 ; 95 \% \mathrm{CI}=0.22-0.773)$ and chemotherapy regimens $(\mathrm{P}=0.042 ; \mathrm{OR}=2.257 ; 95 \% \mathrm{CI}=1.031-4.942)$ were significant following multivariate analysis. Sex $(\mathrm{P}=0.345)$, CEA prior to treatment $(\mathrm{P}=0.052)$, tumor differentiation $(\mathrm{P}=0.173)$ and tumor circumference $(\mathrm{P}=0.294)$ were not significant. Thus, the percentage change in NLR and chemotherapy regimens were significant predictors of $\mathrm{pCR}$.

Analysis for $p C R$ in the validation cohort. Percentage change of $21.5 \%$ NLR was considered to be optimal to predict pCR events in the training cohort (Fig. 2). To verify the association between the percentage change in NLR and pCR, univariate and multivariate analysis for $\mathrm{pCR}$ were performed for the validation cohort (Table IV). Univariate analysis demonstrated that tumor differentiation, chemotherapy regimens and the percentage change in NLR were associated with pCR. However, only the percentage change in NLR $(\mathrm{P}=0.03$; $\mathrm{OR}=0.337 ; 95 \%$ $\mathrm{CI}=0.126-0.9)$ was significant following multivariate analysis.

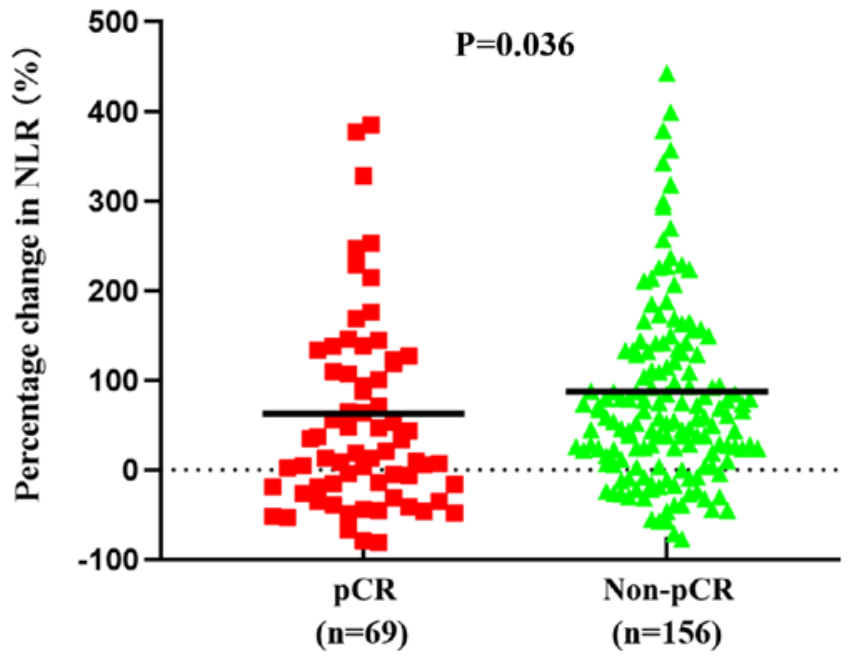

Figure 1. Mean percentage change in NLR values between the pCR and non-pCR groups in the training cohort (bold bar). NLR, neutrophil-toSlymphocyte ratio; $\mathrm{pCR}$, pathological complete response.

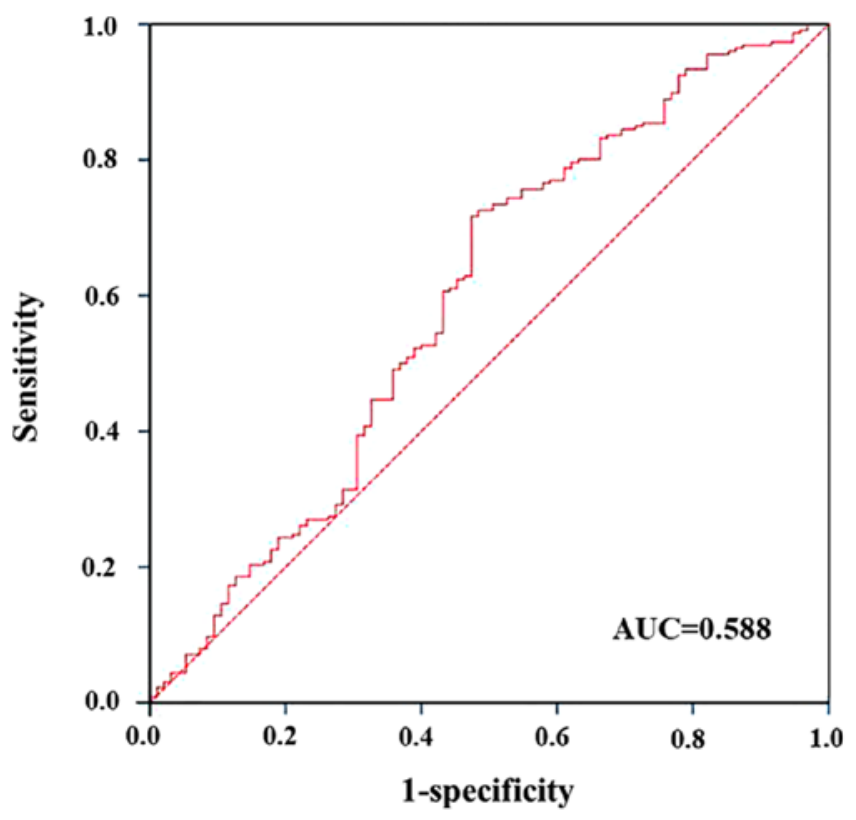

Figure 2. Receiver operator characteristic analysis of the percentage change in neutrophil-to-lymphocyte ratio for prediction of pathological complete response. AUC, area under the curve.

In summary, the results verified that the percentage change in NLR was a significant predictor of $\mathrm{pCR}$.

\section{Discussion}

Systemic inflammatory responses have become a focus of interest for physicians, and cancer-related inflammatory responses have been recognized as a 'hallmark' of cancer development and progression (26). PLR and NLR are important indicators of systemic inflammatory responses $(27,28)$ and the increase in these indicators has been confirmed as adverse factors for the prognosis of multiple malignant tumors, including colon cancer and rectal cancer $(29,30)$. However, the association between systemic inflammatory responses 
Table III. Univariate and multivariate analysis in the training cohort.

\begin{tabular}{|c|c|c|c|c|}
\hline \multirow[b]{2}{*}{ Variable } & \multicolumn{2}{|c|}{ Univariate } & \multicolumn{2}{|c|}{ Multivariate } \\
\hline & OR value $(95 \% \mathrm{CI})$ & P-value & OR value $(95 \% \mathrm{CI})$ & P-value \\
\hline Age ( $\leq 65$ vs. $>65$ years $)$ & $1.14(0.62-2.11)$ & 0.668 & & \\
\hline Sex (male vs. female) & $0.47(0.21-1.08)$ & 0.070 & $1.38(0.71-2.68)$ & 0.345 \\
\hline CEA prior to treatment ( $\leq 5 \mathrm{vs} .>5 \mu \mathrm{g} / \mathrm{l})$ & $0.44(0.24-0.82)$ & 0.009 & $0.53(0.27-1.01)$ & 0.052 \\
\hline Clinical T stage (T2 vs. >T3-4) & $1.08(0.55-2.13)$ & 0.817 & & \\
\hline Clinical N stage (N0 vs. N1-2) & $0.97(0.43-2.17)$ & 0.955 & & \\
\hline Tumor size $(\leq 5 \mathrm{vs} .>5 \mathrm{~cm})$ & $1.42(0.74-2.72)$ & 0.628 & & \\
\hline Tumor circumference ( $\leq 50$ vs. $>50 \%$ ) & $0.59(0.33-1.05)$ & 0.073 & $0.72(0.39-1.33)$ & 0.294 \\
\hline Mesorectal fascia (negative vs. positive) & $1.07(0.59-1.94)$ & 0.832 & & \\
\hline Distance from anal verge $(\leq 5 \mathrm{vs} .>5 \mathrm{~cm})$ & $0.69(0.39-1.23)$ & 0.204 & & \\
\hline Tumor differentiation (low vs. medium, high) & $0.36(0.17-0.90)$ & 0.023 & $0.51(0.19-1.34)$ & 0.173 \\
\hline Operation interval of radiotherapy ( $\leq 7 \mathrm{vs.}>7$ weeks) & $1.36(0.76-2.44)$ & 0.301 & & \\
\hline $\begin{array}{l}\text { Chemotherapy regimens (with oxaliplatin vs. } \\
\text { without oxaliplatin) }\end{array}$ & $3.04(1.44-6.41)$ & 0.003 & $2.26(1.03-4.94)$ & 0.042 \\
\hline Chemotherapy courses ( $\leq 4$ vs. $>4$ ) & $1.91(0.74-4.91)$ & 0.174 & & \\
\hline$\%$ change in NLR $(\leq 21.5$ vs. $>21.5)$ & $0.36(0.20-0.67)$ & 0.001 & $0.41(0.22-0.77)$ & 0.006 \\
\hline
\end{tabular}

CEA, carcino-embryonic antigen; T, tumor; N, lymph node; NLR, neutrophil-to-lymphocyte ratio; OR, odds ratio; CI, confidence interval.

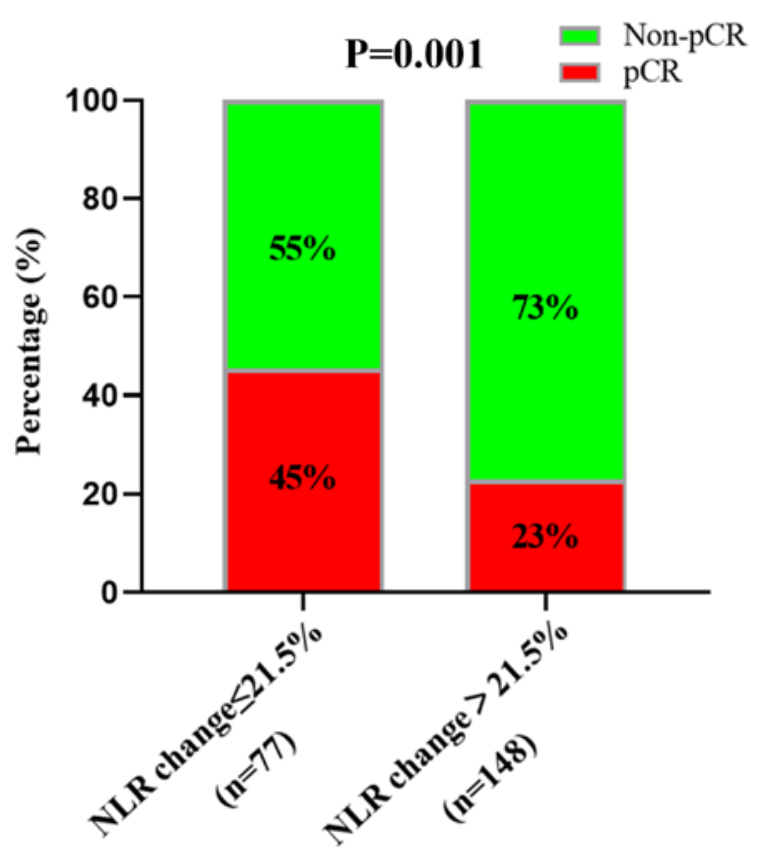

Figure 3. Responsiveness in patients with a percentage change of $\leq 21.5$ and $>21.5 \%$ NLR. NLR, neutrophil-to-lymphocyte ratio; pCR, pathological complete response.

and tumor regression following nCRT in patients with LARC remains unclear. In the current study, pre- and post-nCRT NLR and PLR, and changes in these indices from pre-nCRT to post-nCRT were investigated. Through logistic regression analysis, the results demonstrated that the percentage change in NLR was associated with pCR. These findings were validated by the validation cohort. To the best of our knowledge, the current study is the first to assess the predictive impact of NLR change on tumor treatment outcomes of nCRT in patients with LARC. The results demonstrated that the percentage change in NLR from pre-nCRT to post-nCRT could be used to identify patients achieving pCR following nCRT.

Previous studies have focused on the predictive value of baseline systemic inflammatory responses. Dudani et al (31) reviewed 1,527 patients with rectal cancer receiving nCRT and surgery, and revealed that NLR and PLR were not predictors for disease-free survival or overall survival, and could not predict pCR. Additionally, Shen et al (29) reported that there was no significant association between tumor response and NLR to nCRT. It has previously been reported that baseline systemic inflammatory responses could not predict tumor responses in colorectal cancer $(32,33)$. In the present study, there was no significant association between pCR and NLR or PLR during nCRT.

Furthermore, the results also revealed that adding oxaliplatin to the chemotherapy regimens improved the rate of pCR. Currently, it is controversial whether combining Oxaliplatin with nCRT could improve prognosis and the rate of $\mathrm{pCR}$. The results from the FOWARC study demonstrated that the application of oxaliplatin to nCRT for the treatment of middle and lower rectal cancer exhibited higher rates of pCR (34). Allegra et al (35) compared the rates of $\mathrm{pCR}$ in patients receiving neoadjuvant chemoradiotherapy with or without oxaliplatin, and the results did not indicate any significant differences (17.8 vs. 19.5\%; $\mathrm{P}=0.42$ ), which were inconsistent with the results obtained in the current study. This may be due to the higher dose used in the current study and the FOWARC study compared with that used in other studies (85 vs. $50-60 \mathrm{mg} / \mathrm{m}^{2}$ ) $(34,35)$.

In numerous studies involving rectal cancer, CEA levels were investigated as potential predictors of rectal cancer. Moureau-Zabotto et al (36) demonstrated that a pre-nCRT 
Table IV. Univariate and multivariate analysis in the validation cohort.

\begin{tabular}{|c|c|c|c|c|}
\hline \multirow[b]{2}{*}{ Variable } & \multicolumn{2}{|c|}{ Univariate } & \multicolumn{2}{|c|}{ Multivariate } \\
\hline & OR value $(95 \% \mathrm{CI})$ & P-value & OR value $(95 \% \mathrm{CI})$ & P-value \\
\hline Age ( $\leq 65$ vs. $>65$ years $)$ & $1.80(0.58-5.58)$ & 0.304 & & \\
\hline Sex (male vs. female) & $1.03(0.39-2.73)$ & 0.951 & & \\
\hline CEA prior to treatment ( $\leq 5 \mathrm{vs} .>5 \mu \mathrm{g} / \mathrm{l})$ & $1.64(0.66-4.10)$ & 0.286 & & \\
\hline Clinical T stage (T2 vs. >T3-4) & $1.10(0.36-3.23)$ & 0.862 & & \\
\hline Clinical N stage (N0 vs. N1-2) & $1.75(0.46-6.72)$ & 0.411 & & \\
\hline Tumor size $(\leq 5 \mathrm{vs} .>5 \mathrm{~cm})$ & $0.80(0.31-2.10)$ & 0.650 & & \\
\hline Tumor circumference ( $\leq 50$ vs. $>50 \%$ ) & $0.51(0.20-1.26)$ & 0.140 & & \\
\hline Mesorectal fascia (negative vs. positive) & $1.24(0.48-3.21)$ & 0.665 & & \\
\hline Distance from anal verge ( $\leq 5$ vs. $>5 \mathrm{~cm}$ ) & $0.84(0.33-2.16)$ & 0.722 & & \\
\hline Tumor differentiation (low vs. medium, high) & $0.33(0.08-1.45)$ & 0.023 & $0.32(0.07-1.55)$ & 0.157 \\
\hline Operation interval of radiotherapy ( $\leq 7$ vs. $>7$ weeks) & $1.97(0.70-5.54)$ & 0.194 & & \\
\hline $\begin{array}{l}\text { Chemotherapy regimens (with oxaliplatin vs. } \\
\text { without oxaliplatin) }\end{array}$ & $3.06(0.95-9.87)$ & 0.054 & $2.18(0.64-7.47)$ & 0.213 \\
\hline Chemotherapy course ( $\leq 4$ vs. $>4$ courses) & $1.76(0.53-5.84)$ & 0.348 & & \\
\hline Percentage change in NLR ( $\leq 21.5$ vs. $>21.5 \%$ ) & $0.34(0.13-0.85)$ & 0.019 & $0.34(0.13-0.90)$ & 0.030 \\
\hline
\end{tabular}

CEA, carcino-embryonic antigen; T, tumor; N, lymph node; NLR, neutrophil-to-lymphocyte ratio; OR, odds ratio; CI, confidence interval.

serum CEA level of $<5 \mathrm{ng} / \mathrm{ml}$ was significantly associated with pCR and tumor downstaging (36). However, other studies did not support this conclusion (37). In the current study, a pre-nCRT serum CEA level of $\leq 5 \mathrm{ng} / \mathrm{ml}$ was significantly associated with pCR according to univariate analysis; however, the results of multivariate analysis $(\mathrm{P}=0.052)$ were not significant. This result may be due to the small simple size used in the current study.

The present study had several limitations. Since data was obtained from a single center, the results could not be validated with those from another institute. Furthermore, the retrospective observational design of the current study may cause bias. Further prospective studies are required to validate the results of the present study. The results demonstrated an association between the percentage change in NLR and responsiveness to nCRT among patients with rectal cancer. However, the results failed to determine the cause of this association. Furthermore, a reduction in circulating immunocytes may be due to cytotoxic neoadjuvant chemotherapy. However, neoadjuvant radiation and chemotherapy have been reported to release neoantigens by killing tumor cells, and the immune reaction may cause an increase in circulating immunocytes (38). However, the ratios of different immunocytes as an independent prognostic factor, or parameters associated to therapeutic activity were not examined. Therefore, additional prognostic data is required.

In conclusion, the results of the current study investigated the pre- and post-nCRT systemic inflammatory indices and changes in these indices from pre-nCRT to post-nCRT. The association between the percentage change in NLR and responsiveness to nCRT among patients with rectal cancer was determined, and the results demonstrated that this association could identify patients who achieved pCR following neoadjuvant chemoradiotherapy. These results may provide a novel strategy for colorectal cancer treatment.

\section{Acknowledgements}

Not applicable.

\section{Funding}

The present study was supported by the Fundamental Research Funds for the Central Universities (grant no. 16ykjc25), the Sun Yat-Sen University Clinical Research 5010 Program (grant no. 2016005), the National Health and Medical Research Council Grant (grant no. 1158402) and the Natural Science Foundation of Guangdong Province (grant no. 2018A030313621).

\section{Availability of data and materials}

The datasets used and/or analyzed during the present study are available from the corresponding author on reasonable request.

\section{Authors' contributions}

SCL, LW, JD and LK designed the study. SLL and LH were responsible for data collection. ZL and SCL analyzed data. SLL, LH, JD, LW and LK drafted the manuscript. SCL, JD and LW revised the manuscript. All authors read and approved the final manuscript.

\section{Ethics approval and consent to participate}

The study protocol was reviewed and approved by the Institutional Review Board of the Sixth Affiliated Hospital, Sun Yat-sen University, China. The present study was carried out in accordance with the recommendations of the Declaration 
of Helsinki for biomedical research involving human patients. Written informed consent was obtained from patients prior to enrollment.

\section{Patient consent for publication}

Not applicable.

\section{Competing interests}

The authors declare that they have no competing interests.

\section{References}

1. Brenner H, Kloor M and Pox CP: Colorectal cancer. Lancet 383 : 1490-1502, 2014

2. Benson AB, Venook AP Al-Hawary MM, Cederquist L, Chen YJ Ciombor KK, Cohen S, Cooper HS, Deming D, Engstrom PF, et al: Rectal cancer, version 2.2018, NCCN Clinical Practice Guidelines in Oncology. J Natl Compr Canc Netw 16: 874-901, 2018.

3. Rödel C, Liersch T, Becker H, Fietkau R, Hohenberger W, Hothorn T, Graeven U, Arnold D, Lang-Welzenbach M, Raab HR, et al: Preoperative chemoradiotherapy and postoperative chemotherapy with fluorouracil and oxaliplatin versus fluorouracil alone in locally advanced rectal cancer: Initial results of the German CAO/ARO/AIO-04 randomised phase 3 trial. Lancet Oncol 13: 679-687, 2012.

4. Bryant CL, Lunniss PJ, Knowles CH, Thaha MA and Chan CL: Anterior resection syndrome. Lancet Oncol 13: e403-e408, 2012.

5. Fazio VW, Zutshi M, Remzi FH, Parc Y, Ruppert R, Fürst A Celebrezze J Jr, Galanduik S, Orangio G, Hyman N, et al: A randomized multicenter trial to compare long-term functional outcome, quality of life, and complications of surgical procedures for low rectal cancers. Ann Surg 246: 481-490, 2007.

6. Paun BC, Cassie S, MacLean AR, Dixon E and Buie WD: Postoperative complications following surgery for rectal cancer. Ann Surg 251: 807-818, 2010.

7. Nahas SC, Rizkallah Nahas CS, Sparapan Marques CF, Ribeiro U Jr, Cotti GC, Imperiale AR, Capareli FC, Chih Chen AT, Hoff PM and Cecconello I: Pathologic complete response in rectal cancer: Can we detect it? Lessons learned from a proposed randomized trial of watch-and-wait treatment of rectal cancer. Dis Colon Rectum 59: 255-263, 2016

8. Habr-Gama A, Perez RO, Nadalin W, Sabbaga J, Ribeiro U Jr, Silva e Sousa AH Jr, Campos FG, Kiss DR and Gama-Rodrigues J: Operative versus nonoperative treatment for stage 0 distal rectal cancer following chemoradiation therapy: Long-term results Ann Surg 240: 711-717; discussion 717-718, 2004.

9. van der Valk MJM, Hilling DE, Bastiaannet E, Meershoek-Klein Kranenbarg E, Beets GL, Figueiredo NL, Habr-Gama A, Perez RO, Renehan AG and van de Velde CJH; IWWD Consortium: Long-term outcomes of clinical complete responders after neoadjuvant treatment for rectal cancer in the International Watch \& Wait Database (IWWD): An international multicentre registry study. Lancet 391: 2537-2545, 2018.

10. Habr-Gama A, São Julião GP, Fernandez LM, Vailati BB Andrade A, Araújo SEA, Gama-Rodrigues J and Perez RO: Achieving a complete clinical response After neoadjuvant chemoradiation that does not require surgical resection: It may take longer than you think! Dis Colon Rectum 62: 802-808, 2019

11. Yeom SS, Lee SY, Kim CH, Kim YJ, Nam TK and Kim HR: The outcome of non-operative treatment for rectal cancer patients who had been cCR after neoadjuvant chemoradiotherapy. Asian J Surg 42, 2019.

12. Renehan AG, Malcomson L, Emsley R, Scott N and O'Dwyer ST; OnCoRe project investigators: Watch-and-wait versus surgical resection for patients with rectal cancer-Authors' reply. Lancet Oncol 17: e134-e135, 2016.

13. Kong JC, Guerra GR, Warrier SK, Ramsay RG and Heriot AG: Outcome and salvage surgery following 'watch and wait' for rectal cancer after neoadjuvant therapy: A systematic review. Dis Colon Rectum 60: 335-345, 2017.

14. Malietzis G, Giacometti M, Askari A, Nachiappan S, Kennedy RH, Faiz OD, Aziz O and Jenkins JT: A preoperative neutrophil to lymphocyte ratio of 3 predicts disease-free survival after curative elective colorectal cancer surgery. Ann Surg 260: 287-292, 2014
15. Lee DY, Hong SW, Chang YG, Lee WY and Lee B: Clinical significance of preoperative inflammatory parameters in gastric cancer patients. J Gastric Cancer 13: 111-116, 2013.

16. Stotz M, Gerger A, Eisner F, Szkandera J, Loibner H, Ress AL, Kornprat P, AlZoughbi W, Seggewies FS, Lackner C, et al: Increased neutrophil-lymphocyte ratio is a poor prognostic factor in patients with primary operable and inoperable pancreatic cancer. Br J Cancer 109: 416-421, 2013.

17. Pichler M, Hutterer GC, Stoeckigt C, Chromecki TF, Stojakovic T, Golbeck S, Eberhard K, Gerger A, Mannweiler S, Pummer K and Zigeuner R: Validation of the pre-treatment neutrophillymphocyte ratio as a prognostic factor in a large European cohort of renal cell carcinoma patients. Br J Cancer 108: 901-907, 2013.

18. Azab B, Shah N, Radbel J, Tan P, Bhatt V, Vonfrolio S, Habeshy A, Picon A and Bloom S: Pretreatment neutrophil/lymphocyte ratio is superior to platelet/lymphocyte ratio as a predictor of long-term mortality in breast cancer patients. Med Oncol 30: 432, 2013.

19. Yamada S, Fujii T, Yabusaki N, Murotani K, Iwata N, Kanda M, Tanaka C, Nakayama G, Sugimoto H, Koike M, et al: Clinical implication of inflammation-based prognostic score in pancreatic cancer: Glasgow prognostic score is the most reliable parameter. Medicine (Baltimore) 95: e3582, 2016.

20. Li MX, Liu XM, Zhang XF, Zhang JF, Wang WL, Zhu Y, Dong J, Cheng JW, Liu ZW, Ma L and Lv Y: Prognostic role of neutrophil-to-lymphocyte ratio in colorectal cancer: A systematic review and meta-analysis. Int J Cancer 134: 2403-2413, 2014.

21. Roxburgh CS, Salmond JM, Horgan PG, Oien KA and McMillan DC: The relationship between the local and systemic inflammatory responses and survival in patients undergoing curative surgery for colon and rectal cancers. J Gastrointest Surg 13: 2011-2019, 2009.

22. Heo J, Oh YT, Noh OK, Chun M, Park JE and Cho SR: Nodal tumor response according to the count of peripheral blood lymphocyte subpopulations during preoperative chemoradiotherapy in locally advanced rectal cancer. Radiat Oncol J 34: 305-312, 2016.

23. Lee IH, Hwang S, Lee SJ, Kang BW, Baek D, Kim HJ, Park SY, Park JS, Choi GS, Kim JC, et al: Systemic inflammatory response after preoperative chemoradiotherapy can affect oncologic outcomes in locally advanced rectal cancer. Anticancer Res 37: 1459-1465, 2017.

24. Laird BJ, Fallon M, Hjermstad MJ, Tuck S, Kaasa S, Klepstad P and McMillan DC: Quality of life in patients with advanced cancer: Differential association with performance status and systemic inflammatory response. J Clin Oncol 34: 2769-2775, 2016.

25. Tong GJ, Zhang GY, Liu J, Zheng ZZ, Chen Y, Niu PP and $\mathrm{Xu} X$ : Comparison of the eighth version of the American Joint Committee on Cancer manual to the seventh version for colorectal cancer: A retrospective review of our data. World J Clin Oncol 9: 148-161, 2018.

26. Hanahan D and Weinberg RA: Hallmarks of cancer: The next generation. Cell 144: 646-674, 2011.

27. Gomez D, Morris-Stiff G, Toogood GJ, Lodge JP and Prasad KR: Impact of systemic inflammation on outcome following resection for intrahepatic cholangiocarcinoma. J Surg Oncol 97: 513-518, 2008.

28. Ku JH, Kang M, Kim HS, Jeong CW, Kwak C and Kim HH: The prognostic value of pretreatment of systemic inflammatory responses in patients with urothelial carcinoma undergoing radical cystectomy. Br J Cancer 112: 461-467, 2015.

29. Shen L, Zhang H, Liang L, Li G, Fan M, Wu Y, Zhu J and Zhang Z: Baseline neutrophil-lymphocyte ratio $(\geq 2.8)$ as a prognostic factor for patients with locally advanced rectal cancer undergoing neoadjuvant chemoradiation. Radiat Oncol 9: 295, 2014

30. Braun LH, Baumann D, Zwirner K, Eipper E, Hauth F, Peter A, Zips D and Gani C: Neutrophil-to-lymphocyte ratio in rectal cancer-novel biomarker of tumor immunogenicity during radiotherapy or confounding variable? Int J Mol Sci 20: 2448, 2019.

31. Dudani S, Marginean H, Tang PA, Monzon JG, Raissouni S, Asmis TR, Goodwin RA, Gotfrit J, Cheung WY and Vickers MM: Neutrophil-to-lymphocyte and platelet-to-lymphocyte ratios as predictive and prognostic markers in patients with locally advanced rectal cancer treated with neoadjuvant chemoradiation. BMC Cancer 19: 664, 2019.

32. Picardo SL, Teo M, Jalil KIA, Naqvi SY,Morris PG, Breathnach OS, Grogan W, Leonard GD and Hennessy B: Correlation between platelet/lymphocyte ratio, neutrophil/lymphocyte ratio and response to neoadjuvant chemoradiation therapy in rectal cancer. J Clin Oncol 34: e15140, 2016. 
33. Shin US, You YN, Price BA, Rodriguez-Bigas MA, Skibber JM, Maru DM, Taggart MW, Kopetz S, Eng C, Minsky BD, et al: Is the neutrophil-lymphocyte ratio (NLR) a predictive and prognostic factor in rectal cancer patients treated with neoadjuvant chemoradiation (nCRT)? J Clin Oncol 34: 3605, 2016.

34. Deng Y, Chi P, Lan P, Wang L, Chen W, Cui L, Chen D, Cao J, Wei H, Peng X, et al: Modified FOLFOX6 with or without radiation versus fluorouracil and leucovorin with radiation in neoadjuvant treatment of locally advanced rectal cancer: Initial results of the Chinese FOWARC multicenter, open-label, randomized three-arm phase III trial. J Clin Oncol 34: 3300-3307, 2016.

35. Allegra CJ, Yothers G, O'Connell MJ, Beart RW, Wozniak TF, Pitot HC, Shields AF, Landry JC, Ryan DP, Arora A, et al: Neoadjuvant 5-FU or capecitabine plus radiation with or without oxaliplatin in rectal cancer patients: A phase III randomized clinical trial. J Natl Cancer Inst 107: djv248, 2015.
36. Moureau-Zabotto L, Farnault B, de Chaisemartin C, Esterni B, Lelong B, Viret F, Giovannini M, Monges G, Delpero JR, Bories E, et al: Predictive factors of tumor response after neoadjuvant chemoradiation for locally advanced rectal cancer. Int J Radiat Oncol Biol Phys 80: 483-491, 2011.

37. Kalady MF, de Campos-Lobato LF, Stocchi L, Geisler DP, Dietz D, Lavery IC and Fazio VW: Predictive factors of pathologic complete response after neoadjuvant chemoradiation for rectal cancer. Ann Surg 250: 582-589, 2009.

38. Burnette B, Fu YX and Weichselbaum RR: The confluence of radiotherapy and immunotherapy. Front Oncol 2: 143, 2012.

This work is licensed under a Creative Commons Attribution-NonCommercial-NoDerivatives 4.0 International (CC BY-NC-ND 4.0) License. 